\title{
Florística, estrutura e características do solo de um fragmento de floresta paludosa no sudeste do Brasil
}

\author{
Laércio Loures ${ }^{1}$, Douglas Antônio de Carvalhoo, 2, Evandro Luiz Mendonça Machado ${ }^{3}$ e \\ João José Granate de Sá e Melo Marques ${ }^{4}$
}

Recebido em 13/07/2006. Aceito em 8/03/2007

\begin{abstract}
RESUMO - (Florística, estrutura e características do solo de um fragmento de floresta paludosa no sudeste do Brasil). Realizou-se o levantamento da comunidade arbórea de uma floresta semidecidual montana ribeirinha, situada no alto Rio Pardo, Santa Rita de Caldas, Minas Gerais (altitude 1.156 a $1.203 \mathrm{~m}$ ), com o objetivo de avaliar as variações estruturais e variáveis ambientais relacionadas ao substrato. Foram analisadas a densidade, área basal e distribuição de tamanho das árvores e a composição florística da comunidade. Foram alocadas 25 parcelas de $10 \times 40 \mathrm{~m}$ para amostragem dos indivíduos arbóreos com diâmetro à altura do peito $\geq 5 \mathrm{~cm}$. Também foram coletados dados topográficos e amostras do solo para análises químicas e texturais. Foram registrados 2.982 indivíduos, 50 famílias, 78 gêneros e 110 espécies. Foram verificados três subgrupos de solos (Organossolos, Gleissolos Melânicos e Gleissolos Háplicos), distribuídos em sequiência, acompanhando o curso do riacho. A comunidade arbórea apresentou uma composição florística e estrutural diferente das florestas paludosas e aluviais do Sudeste e Sul do Brasil, em altitudes menores. Uma análise de correspondência canônica detectou um gradiente de distribuição das espécies arbóreas, principalmente correlacionadas com as variações de argila e de cota altimétrica.
\end{abstract}

Palavras-chave: Floresta paludosa montana, floresta ciliar, floresta ribeirinha, solo

\begin{abstract}
Floristics, structure and soil characteristics of a swamp forest fragment, in southeast Brazil). A survey of the tree community was conducted for an upland semideciduous riparian forest, located on the upper Rio Pardo, Santa Rita de Caldas, Minas Gerais (1156 to $1203 \mathrm{~m}$ altitude), in order to evaluate structural variation and environmental variables associated with the substrate. Density, basal area, tree size distribution and floristic composition were evaluated. In $2510 \times 40 \mathrm{~m}$ plots, all trees with dbh $\geq 5 \mathrm{~cm}$ were sampled, topographical aspects were determined and soil samples were collected for chemical and textural analyses. A total of 2982 individuals from 50 families, 78 genera and 110 species were recorded. A sequence of three soil subgroups (Organosoil, Melanic Gleisoil and Haplic Gleisoil) was registered in a downstream direction. Floristic composition and structure the tree community was different from other swamp and alluvial forests of southern and southeastern Brazil, at lower altitudes. Canonical correspondence analysis detected a tree-species distribution gradient correlated mainly with clay content and elevation variation.
\end{abstract}

Key words: montane swamp forest, riparian forest, soil

\section{Introdução}

As florestas ripárias funcionam como reguladoras de processos erosivos, estabilizam margens, promovem ciclagem de nutrientes, freiam a carreação de sedimentos para o meio aquático, facilitam a interação solo-água-fauna e promovem a estabilidade térmica dos corpos d'água, entre outras funções (Lima 1989, Pedralli \& Teixeira 1997). Apesar de sua importância, as florestas ripárias aproximam-se da erradicação em várias partes do Brasil (Gibbs et al. 1980). As florestas ripárias são divididas em permanentemente inundada ou paludosa, inundada anualmente ou de várzea e floresta seca (Rodrigues \& Shepherd 1993). As florestas paludosas ou de brejos são naturalmente fragmentadas (Torres et al. 1994, Ivanauskas et al. 1997, Toniato et al. 1998, Paschoal \& Cavassan 1999), ocorrem sobre solos hidromórficos e possuem baixa diversidade (Leitão Filho 1982), apresentando espécies capazes de germinar e crescer em condições de saturação hídrica e conseqüente falta de oxigênio (Joly 1986). As espécies ali ocorrentes podem ser

\footnotetext{
Escola Agrotécnica Federal de Inconfidentes, 37575-000 Inconfidentes, MG, Brasil

2 Universidade Federal de Lavras, Departamento de Biologia, 37200-000 Lavras, MG, Brasil

3 Universidade Federal de Lavras, Departamento de Ciências Florestais, 37200-000 Lavras, MG, Brasil

${ }^{4}$ Universidade Federal de Lavras, Departamento de Ciências do Solo, 37200-000 Lavras, MG, Brasil

5 Autor para correspondência: douglasc@ufla.br
} 
886 Loures, Carvalho, Machado \& Marques: Florística, estrutura e características do solo de um fragmento de floresta...

classificadas em peculiares (exclusivas e não exclusivas) e complementares (de áreas secas e indiferentes) (Torres et al. 1992). As espécies peculiares exclusivas são aquelas restritas às áreas paludosas; as peculiares não exclusivas podem ocorrer em outros ambientes, mas só se destacam em áreas permanentemente inundáveis. As espécies complementares de terras secas se destacam em terras temporariamente inundáveis, mas, nunca nas paludosas; as complementares indiferentes, são aquelas que podem se destacar em qualquer ambiente, florestas paludosas, de galeria, ciliares e de planalto. As florestas paludosas são muito pouco estudadas no Brasil (Toniato et al. 1998), limitando quase que somente ao estado de São Paulo, onde essas formações encontram-se muito degradadas (Torres et al. 1994). Em Minas Gerais, um único estudo foi desenvolvido, no Sul do estado, por Rocha et al. (2005), num continuum de floresta paludosa e de encosta. Este trabalho tem como objetivo estudar a florística e a estrutura da comunidade arbórea de um fragmento de floresta paludosa no alto Rio Pardo, no município de Santa Rita de Caldas, Sul de Minas Gerais, bem como as correlações entre as variações da estrutura dessa comunidade arbórea e as variáveis ambientais relacionadas ao substrato. $\mathrm{O}$ presente estudo é o primeiro em áreas paludosas acima de $1.000 \mathrm{~m}$ no Brasil.

\section{Material e métodos}

O fragmento de floresta paludosa localiza-se no município de Santa Rita de Caldas, MG, no bairro rural do Pião, a $10 \mathrm{~km}$ da sede do município (46²1'20"W; $22^{\circ} 05^{\prime} 44^{\prime \prime}$; ; altitude de 1.158 a $1.204 \mathrm{~m}$ ). O fragmento, com área de 10,5 ha, corresponde a uma floresta estacional semidecidual montana (Veloso et al. 1991) e acompanha o riacho Luiz Espanhol, desde suas nascentes até uma represa artificial, com uma extensão de $800 \mathrm{~m}$. As águas percorrem aproximadamente $300 \mathrm{~m}$ e se encontram com as águas do ribeirão da Prata, que desagua no ribeirão São Bento e este no rio Pardo, próximo à cidade de Santa Rita de Caldas (Fig. 1). O clima é subtropical úmido (Cfb de Köppen) e apresenta condições mesotérmicas úmidas, verões temperados e chuvosos e invernos sujeitos a geadas (INDI 1977). A pluviosidade média anual é de $1.695 \mathrm{~mm}$, com período chuvoso de outubro a março, e seco de julho a agosto; a temperatura média anual é de $7,4{ }^{\circ} \mathrm{C}$ para as mínimas e $25,9^{\circ} \mathrm{C}$ para as máximas; a umidade relativa anual média é de $78,2 \%$.

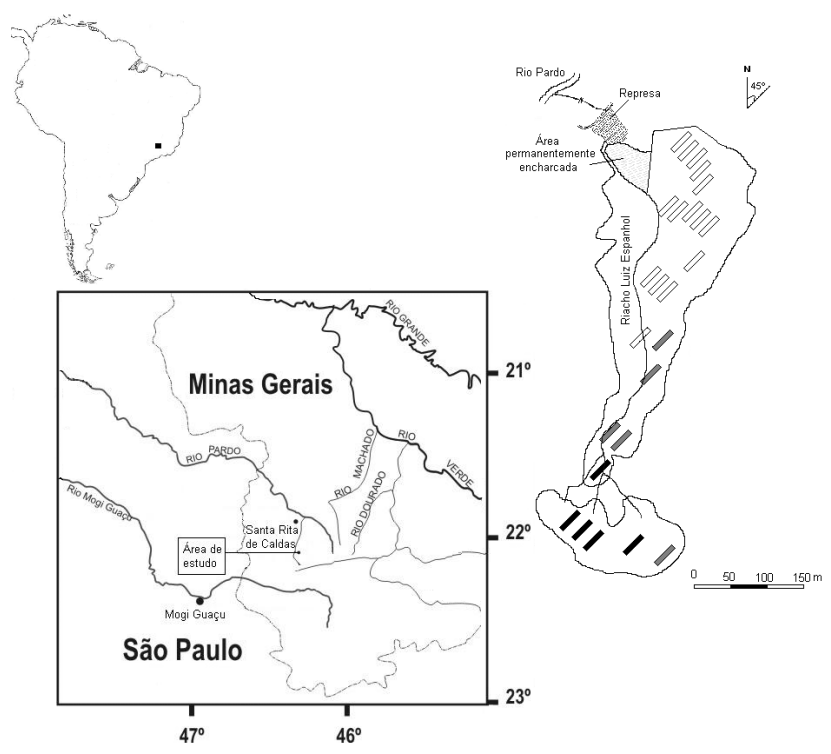

Figura 1. Localização do fragmento de floresta estacional semidecidual montana ribeirinha no alto Rio Pardo, Santa Rita de Caldas, Minas Gerais, e das 25 parcelas de $10 \times 40 \mathrm{~m}$, com suas respectivas classes de solos. $(\boldsymbol{\square}=$ Organossolos; $\square=$ Gleissolos Melânicos; $\square=$ Gleissolos Háplicos).

A vegetação da região é considerada de abrangência do domínio Atlântico (IBGE 1997). O terreno que contorna o fragmento foi cultivado nos últimos 30 anos em sistema de rodízio com pastagem, batata e milho. Devido ao cultivo e, principalmente, às operações mecânicas de colheita da batata, houve danos erosivos ao solo e o assoreamento de algumas áreas. Outras perturbações foram a erosão superficial de alguns trechos da floresta e a erosão em ravina, em canais de escoamento de água, o que provocou espontaneamente a formação de um dique e o levantamento do nível da água em uma área de baixada de aproximadamente $300 \mathrm{~m}^{2}$, em que ocorreu a morte da maioria das árvores atingidas (observações pessoais). Segundo os moradores da região, proprietários e ex-proprietários da área do fragmento, nos últimos sessenta anos não houve mudanças aparentes na mata. Entretanto, há sinais de perturbações como retirada de madeiras, ocorrências de fogo e presença de gado. Ocorrem no fragmento espécies não arbóreas e regionalmente tidas como indicadoras de floresta original, como Canistrum cyatiformis Mez. (Bromeliaceae) e Bambusa taguara Nees (Poaceae). A flora herbácea terrestre do fragmento tem vários representantes típicos de formações paludosas, como Blechnum brasiliense Desv., Echinodorus grandiflorus (Cham. \& Schldtl.) Micheli, Edychium coronarius J. Koering, Piper aduncum L., Pothomorphe umbellata (L.) Miq., 
Eriocaulon decangulare L., Equisetum martii Milde e Eryngium horridum Malme.

A comunidade arbórea foi amostrada em 25 parcelas de 10×40 m, distribuídas com mínimo de $10 \mathrm{~m}$ de distância entre elas, alinhadas no sentido longitudinal, num ângulo de $45^{\circ} \mathrm{NE}$ (Fig. 1). A topografia foi registrada com auxílio de teodolito, altímetro, clinômetro e trena. O solo predominante em cada parcela foi classificado de acordo com o Sistema Brasileiro de Classificação de Solos (EMBRAPA 1999). Em cada parcela, foi coletada uma amostra composta do solo superficial $(0-20 \mathrm{~cm})$, com cerca de $500 \mathrm{~g}$, constituída de cinco sub-amostras (nos vértices e no centro das parcelas). Efetuaram-se análises químicas e texturais das amostras de solo nos Laboratórios de Análises de Solos da Escola Agrotécnica Federal de Inconfidentes e da Universidade Federal de Lavras, seguindo o protocolo da EMBRAPA (1997). As variáveis de solo obtidas foram: $\mathrm{pH}$; teores de $\mathrm{P}, \mathrm{K}, \mathrm{Ca}, \mathrm{Mg}, \mathrm{Al}$; saturação por bases; matéria orgânica e teores de areia, silte e argila.

Nas parcelas foram registrados todos os indivíduos arbóreos vivos com diâmetro à altura do peito (DAP) $\geq 5,0 \mathrm{~cm}$. Os indivíduos com caules divididos desde a base foram incluídos no levantamento quando a área basal total dos caules foi $\geq 19,64 \mathrm{~cm}^{2}$. Para cada indivíduo foram registrados a espécie, DAP (obtido a partir da circunferência), altura total, inclinação do caule e ocorrência de perfilhamento. Também o número de indivíduos mortos foi anotado. Foram coletadas amostras de material botânico das árvores registradas para posterior identificação e inclusão no Herbário ESAL, Universidade Federal de Lavras. A identificação do material botânico foi realizada através de comparações com material de coleções botânicas e de consultas à literatura e a especialistas. As identificações das angiospermas seguem o sistema Angiosperm Phylogeny Group II (APG II 2003).

Para descrever a estrutura da comunidade foram utilizadas as seguintes variáveis, calculadas para cada espécie: altura máxima, número de indivíduos, número de parcelas com ocorrência da espécie, área basal e valor de importância (VI), este último obtido da soma dos valores relativos de número de indivíduos, área basal e frequiência (Martins 1991). Para a determinação da diversidade de espécies foram calculados os índices de Shannon $\left(H^{\prime}\right)$ e de equabilidade de Pielou $(J)$ em base logarítmica natural (Brower \& Zar 1984). Foram preparadas distribuições de densidade de árvores por classes de diâmetro e de altura para os três hábitats de solo, empregando intervalos com amplitudes crescentes para compensar o decréscimo da densidade nas classes de tamanhos maiores (Botrel et al. 2002). Foi adotada a densidade de árvores por hectare ao invés da frequiência por classes porque os habitas de solo tinham áreas desiguais. Para verificar se a distribuição das freqüências de indivíduos arbóreos nas classes de diâmetro e altura era independente do hábitat de solo foi aplicado o teste de qui-quadrado para tabelas de contingência (Zar 1996). Para analisar as correlações entre os gradientes ambientais e vegetacionais, foi empregada a análise de correspondência canônica (ter Braak 1987) sendo esta ordenação indicada para a visualização de relações estreitas entre variáveis ambientais e abundância de espécies (Kent \& Coker 1992). Espécies com menos de 10 indivíduos foram retiradas da matriz de abundância. Retiraram-se das análises todas as variáveis ambientais com correlação aos eixos da ordenação inferior a 0,4 ou com alta redundância conforme Oliveira Filho \& Ratter (1994), restando quatro variáveis: argila, cota, desnível e concentração de Ca.

\section{Resultados}

Variáveis topográficas e edáficas - As três classes de solos identificadas no fragmento florestal (Fig. 1) foram: (a) os Organossolos, situados na parte mais elevada do fragmento, de textura média (20\% das parcelas); (b) os Gleissolos Melânicos localizados na parte intermediária do fragmento, de textura média (20\% das parcelas) e (c) os Gleissolos Háplicos, encontrados no centro e nas áreas mais baixas do fragmento, de textura predominantemente argilosa (60\% das parcelas). As classes de solo diferiram quanto às variáveis químicas, texturais e topográficas (Tab. 1). A principal diferença entre as três classes de solo é o teor de matéria orgânica. A grande quantidade de matéria orgânica nos Organossolos levou a níveis mais baixos de $\mathrm{pH}$ e a uma acidez potencial $(\mathrm{H}+\mathrm{Al})$ mais elevada, embora com teores de nutrientes $(\mathrm{Ca}$ e $\mathrm{Mg}$ ) relativamente altos. Como seria esperado, a argila seguiu comportamento oposto ao da matéria orgânica, sendo mais abundante nos Gleissolos Háplicos. Aparentemente, os Gleissolos Melânicos apresentaram um balanço ideal entre argila e matéria orgânica, com altos teores de nutrientes sem, contudo, apresentarem os altos níveis de acidez e toxidez dos Organossolos. A diferença da cota média da parcela mais alta (cabeceira) e a parcela mais baixa (próximo à represa) foi de $46 \mathrm{~m}$. 
888 Loures, Carvalho, Machado \& Marques: Florística, estrutura e características do solo de um fragmento de floresta...

Tabela 1. Variáveis químicas e texturais das amostras de solo superficial $(0-20 \mathrm{~cm}$ de profundidade), em 1 ha de floresta estacional semidecidual montana ribeirinha, Santa Rita de Caldas, Minas Gerais. Os valores são médias \pm desvio padrão das n amostras de cada uma das três classes de solos: Gleissolos Háplicos, Gleisssolos Melânicos e Organossolos. Testes de $F$ indicaram diferenças significativas entre os três tipos de solos. As médias seguidas de letras diferentes são significativamente diferentes (ANOVA e Tukey-Kramer $a$ posteriori) $\left({ }^{*} p<0,05 ;{ }^{* *} p<0,01 ;{ }^{\text {ns }}\right.$ ño significativo).

\begin{tabular}{|c|c|c|c|c|c|c|}
\hline \multirow{2}{*}{$\begin{array}{l}\text { Variáveis de solo } \\
\text { Cota média }^{1}(\mathrm{~m})\end{array}$} & $\begin{array}{l}\text { Gleissolos Háplicos } \\
\qquad \mathrm{n}=15\end{array}$ & $\begin{array}{l}\text { Gleissolos Melânicos } \\
\qquad n=5\end{array}$ & \multicolumn{3}{|c|}{$\begin{array}{c}\text { Organossolos } \\
n=5\end{array}$} & \multirow{2}{*}{$\begin{array}{c}F \quad p \\
33,02^{* * *}\end{array}$} \\
\hline & $164,00 \pm 6,13^{b}$ & $183,96 \pm 11,99$ a & 193,60 & $\pm 7,13$ & a & \\
\hline Desnível (m) & $1,33 \pm$ & $4,26 \pm 3,29$ & 0,50 & $\pm 1,41$ & & $1,88^{\mathrm{ns}}$ \\
\hline $\mathrm{pH} \mathrm{em} \mathrm{H}_{2} \mathrm{O}$ & $5,55 \pm$ & $5,64 \pm 0,05$ & 5,38 & $3 \pm 0,11$ & $\mathrm{~b}$ & $4,90 *$ \\
\hline P - Mehlich (mg.dm $\left.{ }^{-3}\right)$ & $7,03 \pm 2,57$ & $9,24 \pm 3,24$ & 8,34 & $4 \pm 1,14$ & & $1,63^{\mathrm{ns}}$ \\
\hline $\mathrm{K}^{+}\left(\mathrm{mg} \cdot \mathrm{dm}^{-3}\right)$ & $110,47 \pm 33,10$ & $127,80 \pm 42,58$ & 128,80 & $\pm 40,68$ & & $0,72^{\mathrm{ns}}$ \\
\hline $\mathrm{Ca}^{++}\left(\mathrm{cmolc} \cdot \mathrm{dm}^{-3}\right)$ & $1,42 \pm 0,50^{\mathrm{b}}$ & $2,96 \pm 1,15$ & 2,06 & $5 \pm 0,52$ & $\mathrm{~b}$ & $10,27^{* * *}$ \\
\hline $\mathrm{Mg}^{++}\left(\mathrm{cmolc} \cdot \mathrm{dm}^{-3}\right)$ & $0,82 \pm$ & $1,54 \pm 0,56$ a & 0,96 & $5 \pm 0,27$ & $\mathrm{~b}$ & $6,90^{* *}$ \\
\hline $\mathrm{Al}$ & $0,25 \pm$ & $0,22 \pm 0,04$ & 0,32 & $\pm 0,16$ & & $1,06^{\mathrm{ns}}$ \\
\hline $\mathrm{H}+\mathrm{Al}$ & $9,27 \pm$ & $9,13 \pm 1,20 \mathrm{~b}$ & 13,73 & $\pm 3,00$ & a & 6,12 ** \\
\hline Soma de bases $\left(\mathrm{cmolc}^{\left.-\mathrm{dm}^{-3}\right)}\right.$ & $2,53 \pm$ & $4,84 \pm 1,74$ a & 3,42 & $2 \pm 0,72$ & ab & $8,99^{* *}$ \\
\hline $\begin{array}{l}\mathrm{t}-\text { Capacidade de Troca } \\
\text { Catiônica }\left(\mathrm{cmolc}^{\left.-\mathrm{dm}^{-3}\right)}\right.\end{array}$ & $11,81 \pm$ & $13,92 \pm 2,29$ ab & 17,10 & $\pm 2,39$ & a & $7,72 * *$ \\
\hline $\mathrm{V}$ - saturação por bases $(\%)$ & $21,95 \pm 7,97^{\mathrm{b}}$ & $33,76 \pm 8,60^{a}$ & 20,28 & $\pm 6,46$ & $\mathrm{~b}$ & $4,95^{*}$ \\
\hline m - saturação por alumínio (\%) & $9,71 \pm 4,12$ & $4,88 \pm 2,14$ & 9,34 & $4 \pm 6,03$ & & $2,47 \mathrm{~ns}$ \\
\hline Matéria Orgânica (dag.kg $\left.{ }^{-1}\right)$ & $48,22 \pm 17,60^{b}$ & $53,53 \pm 14,80 \mathrm{~b}$ & 76,23 & $3 \pm 11,60$ & a & $5,61 *$ \\
\hline Areia $(\%)$ & $29,53 \pm 8,85$ & $37,20 \pm 5,81$ & 23,40 & $\pm 16,07$ & & $2,33^{\mathrm{ns}}$ \\
\hline Silte $(\%)$ & $31,93 \pm 12,30^{\mathrm{b}}$ & $28,80 \pm 8,23$ b & 49,60 & $\pm 14,26$ & a & $4,81 *$ \\
\hline Argila (\%) & $38,53 \pm 8,33^{a}$ & $34,00 \pm 3,08$ ab & 27,00 & $\pm 5,24$ & $\mathrm{~b}$ & $5,01 *$ \\
\hline
\end{tabular}

${ }^{1}$ Para altitude, acrescentar $1.000 \mathrm{~m}$

Florística, estrutura e diversidade da comunidade arbórea - Foram identificadas 110 espécies, pertencentes a 50 famílias e 78 gêneros, sendo 105 espécies de angiospermas, quatro espécies de Pteridófitas e uma espécie de Gimnosperma (Tab. 2). As dez famílias que apresentaram maior riqueza foram Myrtaceae (12 espécies), Lauraceae (11), Euphorbiaceae (7), Salicaceae (5), Solanaceae (4), Asteraceae (4), Cyathaceae (3), Fabaceae Faboideae (3), Malvaceae (3) e Myrsinaceae (3), com 70,51\% do total de espécies. Vinte e oito famílias apresentaram somente uma espécie. Os gêneros com mais espécies foram Ocotea (8), Casearia, Solanum (4), Ilex, Sebastiania, Machaerium, Eugenia e Myrcia (3).

Do total de indivíduos (2.982), as famílias com maior abundância foram Myrtaceae, Mysinaceae (24\%), Euphorbiaceae (13\%), Lauraceae (7\%), Cardiopteridaceae (6\%), Aquifoliaceae $(5 \%)$, Meliaceae (4\%), Bignoniaceae, Sapindaceae (3\%), Styracaceae (2\%). As dez espécies com maior número de indivíduos foram Myrsine leuconeura (23\%), Myceugenia ovata (13\%), Sebastiania brasiliensis (12\%), Myrcia laruotteana (7\%), Citronella gongonha (6\%), Nectandra nitidula (5\%), Ilex brasiliensis (4\%), Cedrela odorata (4\%), Tabebuia umbellata (3\%), Styrax leprosum (2\%). Os dez maiores valores de área basal (AB), em $\mathrm{m}^{2}$, foram anotados para Sebastiania brasiliensis $(4,80)$, Myrsine intermedia $(4,44)$, Citronella gongonha $(4,29)$, Cedrela odorata $(3,05)$, Myrceugenia ovata $(2,07)$, Nectandra nitidula (1,73), Tabebuia umbellata $(1,63)$, Myrcia laruotteana (1,30), Ilex brasiliensis $(0,80)$ e Matayba guianensis $(0,79)$. Os maiores VI (valor de importância) foram para Myrsine leuconeura $(37,66)$, Sebastiania brasiliensis $(27,25)$, Myrceugenia ovata (22,61), Citronella gongonha $(19,37)$, Myrcia laruotteana $(14,68)$, Cedrela odorata $(14,47)$, Nectandra nitidula $(13,29)$, Tabebuia umbellata (9,08), Matayba guianensis $(6,00)$ e Styrax leprosum $(5,84)$ (Tab. 2). Ocorreram 338 indivíduos perfilhados ou enforquilhados no nível da superfície do solo (11\%), 729 indivíduos inclinados (24\%) e 246 árvores mortas.

Distribuições em diâmetro e altura - Os Organossolos apresentaram maior densidade na primeira e segunda classes de diâmetro; na quarta classe, ocorreram mais indivíduos nos G. Melânicos (Fig. 2A). Quanto à distribuição em classes de altura, a maior parte dos indivíduos concentrou-se na segunda classe nos três tipos de solos (Fig. 2B). Os Organossolos tiveram maior densidade na terceira classe, que os demais tipos de 
Tabela 2. Espécies arbóreas amostradas em 1 ha de floresta estacional semidecidual montana ribeirinha, Santa Rita de Caldas, Minas Gerais, com seus números de registros (Rg) no Herbário ESAL e parâmetros fitossociológicos: h máx = altura máxima; ni = número de indivíduos; $\mathrm{p}$ = número de parcelas em que ocorreu a espécie; $\mathrm{AB}$ = área basal; VI = valor de importância.

\begin{tabular}{|c|c|c|c|c|c|c|}
\hline Famílias/Espécies & $\operatorname{Rg}$ & $\begin{array}{l}\text { h máx } \\
\text { (m) }\end{array}$ & ni & $\mathrm{p}$ & $\begin{array}{l}\text { AB } \\
\left(\mathrm{m}^{2}\right)\end{array}$ & $\begin{array}{l}\text { VI } \\
\%\end{array}$ \\
\hline \multicolumn{7}{|l|}{ PTERIDOPHYTA } \\
\hline \multicolumn{7}{|l|}{ CYATHEACEAE } \\
\hline Alsophila sternbergii (Sternb.) Conant & 20331 & 3 & 1 & 1 & 0,0154 & 0,26 \\
\hline Cyathea corcovadensis (Raddi) Domin & 20333 & 3 & 1 & 1 & 0,0053 & 0,24 \\
\hline C. phalerata Mart. & 20332 & 1,5 & 9 & 3 & 0,0869 & 1,08 \\
\hline \multicolumn{7}{|l|}{ DICKSONIACEAE } \\
\hline Dicksonia sellowiana (Presl) Hooker & 20334 & 3 & 14 & 9 & 0,2345 & 2,75 \\
\hline \multicolumn{7}{|l|}{ GIMNNOSPERMAE } \\
\hline \multicolumn{7}{|l|}{ ARAUCARIACEAE } \\
\hline Araucaria angustifolia (Bert.) Kuntze & 20319 & 28 & 2 & 2 & 0,1484 & 0,79 \\
\hline \multicolumn{7}{|l|}{ ANGIOSPERMAE } \\
\hline \multicolumn{7}{|l|}{ ANACARDIACEAE } \\
\hline Lithraea molleoides (Vell.) Engler & 20309 & 15 & 4 & 4 & 0,1567 & 1,26 \\
\hline Schinus terebinthifolius Raddi & 20310 & 7 & 3 & 3 & 0,0119 & 0,71 \\
\hline Tapirira obtusa (Benth.) Mitchell & 20312 & 20 & 27 & 6 & 0,3320 & 2,81 \\
\hline \multicolumn{7}{|l|}{ ANNONACEAE } \\
\hline Rollinia emarginata Schltdl. & 20311 & 15 & 10 & 8 & 0,0569 & 2,03 \\
\hline \multicolumn{7}{|l|}{ AQUIFOLIACEAE } \\
\hline Ilex brasiliensis (Sprengel) Loes. & 20314 & 18 & 130 & 19 & 0,8034 & 9,86 \\
\hline I. amara D.C. & 20315 & 11 & 1 & 1 & 0,0048 & 0,24 \\
\hline I. paraguariensis A. St.-Hil. & 20314 & 12 & 20 & 8 & 0,0672 & 2,39 \\
\hline \multicolumn{7}{|l|}{ ARACEAE } \\
\hline Philodendron brasiliensis England & 20317 & 1,5 & 3 & 2 & 0,0170 & 0,53 \\
\hline \multicolumn{7}{|l|}{ ARALIACEAE } \\
\hline Dendropanax cuneatus (DC.) Decne \& Planchon & 20318 & 25 & 44 & 11 & 0,4217 & 4,56 \\
\hline \multicolumn{7}{|l|}{ ARECAEAE } \\
\hline Syagrus romanzoffiana (Cham.) Glassman & 20320 & 20 & 12 & 8 & 0,3589 & 2,76 \\
\hline \multicolumn{7}{|l|}{ ASTERACEAE } \\
\hline Baccharis brachylaenoides DC. & 20321 & 13 & 5 & 2 & 0,0433 & 0,66 \\
\hline Eupatorium morifolium Mill & 20322 & 10 & 1 & 1 & 0,0049 & 0,24 \\
\hline Piptocarpha macropoda Baker & 20323 & 15 & 11 & 3 & 0,0925 & 1,16 \\
\hline Vernonanthura diffusa (Less.) H. Robinson & 20324 & 20 & 1 & 1 & 0,0446 & 0,33 \\
\hline \multicolumn{7}{|l|}{ BIGNONIACEAE } \\
\hline Tabebuia umbellata (Sond.) Sandwith & 20326 & 28 & 88 & 13 & 1,6259 & 9,08 \\
\hline \multicolumn{7}{|l|}{ BORAGINACEAE } \\
\hline Cordia magnoliifolia Cham. & 20327 & 9 & 1 & 1 & 0,0074 & 0,25 \\
\hline \multicolumn{7}{|l|}{ BURSERACEAE } \\
\hline Protium spruceanum (Benth.) Engler & 20328 & 13 & 17 & 7 & 0,4592 & 2,95 \\
\hline P. widgrenii Engler & 20329 & 12 & 3 & 2 & 0,0433 & 0,59 \\
\hline \multicolumn{7}{|l|}{ CANNABACEAE } \\
\hline Celtis pubescens Sprengel & 20405 & 12 & 2 & 1 & 0,0051 & 0,27 \\
\hline Trema micrantha (L.) Blume & 20406 & 11 & 1 & 1 & 0,0079 & 0,25 \\
\hline \multicolumn{7}{|l|}{ CARDIOPTERIDACEAE } \\
\hline Citronella gongonha (Mart.) Howard & 20330 & 30 & 173 & 21 & 4,2890 & 19,37 \\
\hline \multicolumn{7}{|l|}{ CELASTRACEAE } \\
\hline Maytenus ilicifolia Reissek & 20399 & 5 & 1 & 1 & 0,0021 & 0,23 \\
\hline CLETHRACEAE & & & & & & \\
\hline Clethra scabra Pers. & 20335 & 15 & 1 & 1 & 0,0215 & 0,28 \\
\hline CUNONIACEAE & & & & & & \\
\hline Lamanonia ternata Vell. & 20430 & 4 & 1 & 1 & 0,0022 & 0,23 \\
\hline ERICACEAE & & & & & & \\
\hline Gaylussacia brasiliensis (Spreng.)Meisn. & 20336 & 5 & 1 & 1 & 0,0020 & 0,23 \\
\hline
\end{tabular}


890 Loures, Carvalho, Machado \& Marques: Florística, estrutura e características do solo de um fragmento de floresta...

Tabela 2 (continuação)

\begin{tabular}{|c|c|c|c|c|c|c|}
\hline Famílias/Espécies & $\operatorname{Rg}$ & $\begin{array}{l}\text { h máx } \\
\text { (m) }\end{array}$ & ni & $\mathrm{p}$ & $\begin{array}{l}\mathrm{AB} \\
\left(\mathrm{m}^{2}\right)\end{array}$ & $\begin{array}{l}\mathrm{VI} \\
\%\end{array}$ \\
\hline \multicolumn{7}{|l|}{ EUPHORBIACEAE } \\
\hline Alchornea triplinervia (Sprengel) Müll. Arg. & 20337 & 19 & 3 & 2 & 0,0304 & 0,56 \\
\hline Croton floribundus Sprengel & 20338 & 15 & 3 & 1 & 0,0202 & 0,34 \\
\hline C. organensis Baillon & 20339 & 12 & 2 & 1 & 0,0082 & 0,28 \\
\hline Sapium glandulosum (L.) Morong & 20341 & 32 & 14 & 7 & 0,4658 & 2,87 \\
\hline Sebastiania brasiliensis Sprengel & 20342 & 13 & 355 & 24 & 4,8280 & 27,25 \\
\hline S. commersoniana (Baillon) Smith \& Downs & 20343 & 12 & 5 & 4 & 0,0402 & 1,04 \\
\hline S. serrata Muller Argoviensis & 20344 & 8 & 1 & 1 & 0,0037 & 0,24 \\
\hline \multicolumn{7}{|l|}{ FABACEAE FABOIDEAE } \\
\hline Erythrina falcata Bentham & 20346 & 30 & 6 & 3 & 0,5732 & 2,05 \\
\hline Lonchocarpus guillemineanus (Tul.) Malme & 20113 & 32 & 4 & 2 & 0,1416 & 0,84 \\
\hline Machaerium nyctitans (Vell;) Benth. & 20431 & 7 & 1 & 1 & 0,0040 & 0,24 \\
\hline M. minutiflorum Vogel & 20347 & 8 & 1 & 1 & 0,0051 & 0,24 \\
\hline M. villosum Vogel & 20348 & 9 & 1 & 1 & 0,0064 & 0,24 \\
\hline \multicolumn{7}{|l|}{ FABACEAE MIMOSOIDEAE } \\
\hline Inga vulpina Mart. ex. O. Benth & 20349 & 12 & 8 & 4 & 0,0997 & 1,27 \\
\hline \multicolumn{7}{|l|}{ LAURACEAE } \\
\hline Nectandra nitidula Nees & 20354 & 18 & 142 & 24 & 1,7318 & 13,29 \\
\hline N. lanceolata Nees & 20353 & 15 & 2 & 2 & 0,0510 & 0,57 \\
\hline Ocotea aciphylla (Nees) Mez & 20359 & 10 & 1 & 1 & 0,0113 & 0,25 \\
\hline O. diospyrifolia (Meisner) Mez & 20360 & 20 & 4 & 3 & 0,0613 & 0,86 \\
\hline O. elegans $\mathrm{Mez}$ & 20353 & 13 & 5 & 2 & 0,0512 & 0,67 \\
\hline O. indecora (Schott) $\mathrm{Mez}$ & 20363 & 18 & 2 & 1 & 0,0420 & 0,36 \\
\hline O. laxa (Nees) $\mathrm{Mez}$ & 20361 & 13 & 20 & 8 & 0,1510 & 2,57 \\
\hline O. odorífera (Vell.) Rohwer & 20362 & 9 & 2 & 2 & 0,0056 & 0,47 \\
\hline O. pulchella Mart. & 20356 & 14 & 2 & 1 & 0,0209 & 0,31 \\
\hline O. velutina (Nees) Rohwer & 20357 & 16 & 2 & 1 & 0,0473 & 0,37 \\
\hline Persea venosa Nees & 20358 & 18 & 11 & 6 & 0,3798 & 2,38 \\
\hline \multicolumn{7}{|l|}{ LYTHRACEAE } \\
\hline Lafoensia pacari A. St.-Hil. & 20364 & 23 & 3 & 3 & 0,1382 & 0,99 \\
\hline \multicolumn{7}{|l|}{ MAGNOLIACEAE } \\
\hline Talauma ovata A. St.-Hil. & 20365 & 18 & 24 & 9 & 0,2445 & 3,11 \\
\hline \multicolumn{7}{|l|}{ MALVACEAE } \\
\hline Chorisia speciosa St.-Hil. & 20366 & 30 & 1 & 1 & 10,8841 & 24,22 \\
\hline Luehea divaricata Mart. \& Zucc. & 20367 & 30 & 5 & 4 & 0,7471 & 2,60 \\
\hline Pseudobombax grandiflorum (Cav.) A. Robyns & 20368 & 14 & 1 & 1 & 0,0445 & 0,33 \\
\hline \multicolumn{7}{|l|}{ MELASTOMATACEAE } \\
\hline Miconia cinerascens Miq. & 20368 & 11 & 1 & 1 & 0,0032 & 0,24 \\
\hline M. pusilliflora (DC.) Triana & & 13 & 9 & 3 & 0,0655 & 1,03 \\
\hline \multicolumn{7}{|l|}{ MELIACEAE } \\
\hline Cedrela fissilis Vell. & 20371 & 10 & 1 & 1 & 0,0032 & 0,24 \\
\hline C. odorata $\mathrm{L}$. & 20370 & 30 & 108 & 21 & 3,0514 & 14,47 \\
\hline \multicolumn{7}{|l|}{ MORACEAE } \\
\hline Ficus enormis (Mart.) Miq. & 20372 & 18 & 6 & 6 & 0,0505 & 1,49 \\
\hline Sorocea bonplandii (Baillon) W. Burger & 20373 & 10 & 3 & 3 & 0,0144 & 0,72 \\
\hline \multicolumn{7}{|l|}{ MYRSINACEAE } \\
\hline Myrsine leuconeura Mez & 20386 & 20 & 697 & 23 & 4,4354 & 37,66 \\
\hline M. umbellata Mart. & 20375 & 5 & 1 & 1 & 0,0081 & 0,25 \\
\hline M. venosa A.D.C. & 20387 & 16 & 7 & 5 & 0,0822 & 1,40 \\
\hline \multicolumn{7}{|l|}{ MYRTACEAE } \\
\hline Calyptranthes clusifolia O. Berg & 20376 & 14 & 1 & 1 & 0,0112 & 0,25 \\
\hline C. grandifolia $\mathrm{O}$. Berg & 20378 & 15 & 7 & 3 & 0,1033 & 1,05 \\
\hline Eugenia blastantha (O. Berg) D. Legrand & 20379 & 5 & 1 & 1 & 0,0028 & 0,24 \\
\hline E. dodonaeifolia Cambess. & 20380 & 14 & 30 & 10 & 0,1585 & 3,32 \\
\hline Myrceugenia ovata (Hook. \& Arn.) & 20384 & 16 & 392 & 25 & 2,0715 & 22,61 \\
\hline Myrcia laruotteana Cambess. & 20383 & 9 & 218 & 23 & 1,2958 & 14,68 \\
\hline
\end{tabular}


Tabela 2 (continuação)

\begin{tabular}{|c|c|c|c|c|c|c|}
\hline Famílias/Espécies & $\operatorname{Rg}$ & $\begin{array}{l}\text { h máx } \\
\text { (m) }\end{array}$ & ni & $\mathrm{p}$ & $\begin{array}{l}\mathrm{AB} \\
\left(\mathrm{m}^{2}\right)\end{array}$ & $\begin{array}{l}\text { VI } \\
\%\end{array}$ \\
\hline Myrcia splendens (Swartz) DC. & 20382 & 15 & 44 & 14 & 0,2621 & 4,80 \\
\hline M. tomentosa (Aublet) DC. & 20374 & 11 & 4 & 4 & 0,0264 & 0,98 \\
\hline Myrcianthes gigantea Legrant & 20421 & 12 & 5 & 3 & 0,0224 & 0,81 \\
\hline Myrciaria delicatula DC. & 20381 & 23 & 4 & 2 & 0,1130 & 0,78 \\
\hline M. tenella (DC.) O. Berg & 20385 & 9 & 3 & 2 & 0,0060 & 0,51 \\
\hline Neumitranthes warmingiana (Kiaersk.) Mattos & 20433 & 9 & 1 & 1 & 0,0066 & 0,24 \\
\hline \multicolumn{7}{|l|}{ NYCTAGINACEE } \\
\hline Guapira opposita (Vell.) Reitz & 20434 & 7 & 1 & 1 & 0,0023 & 0,23 \\
\hline Pisonia ambigua Heimerl & 20388 & 9 & 1 & 1 & 0,0202 & 0,27 \\
\hline \multicolumn{7}{|l|}{ OCHNACEAE } \\
\hline Ouratea parviflora (DC.) Baillon & 20389 & 9 & 3 & 1 & 0,0126 & 0,32 \\
\hline \multicolumn{7}{|l|}{ OLEACEAE } \\
\hline Chionanthus micranthus (Mart) Lozano \& Fuertes & 20390 & 10 & 3 & 2 & 0,0159 & 0,53 \\
\hline \multicolumn{7}{|l|}{ PHYLLANTHACEAE } \\
\hline Hyeronima ferruginea Müll. Arg. & & 10 & 1 & 1 & 0,0039 & 0,24 \\
\hline \multicolumn{7}{|l|}{ PROTEACEAE } \\
\hline Roupala brasiliensis Klotzsch & 20391 & 23 & 1 & 1 & 0,1233 & 0,50 \\
\hline \multicolumn{7}{|l|}{ ROSACEAE } \\
\hline Prunus myrtifolia (L.) Urban & 20392 & 15 & 8 & 4 & 0,0411 & 1,14 \\
\hline \multicolumn{7}{|l|}{ RUBIACEAE } \\
\hline Guettarda uruguensis Cham. \& Schltdl. & 20435 & 14 & 12 & 4 & 0,2494 & 1,74 \\
\hline \multicolumn{7}{|l|}{ RUSCACEAE } \\
\hline Cordyline spectabilis Couth & 20308 & 5 & 1 & 1 & 0,0040 & 0,24 \\
\hline \multicolumn{7}{|l|}{ SALICACEAE } \\
\hline Casearia arborea (L.C. Rich.) Urban & & 15 & 1 & 1 & 0,0230 & 0,28 \\
\hline C. lasiophylla Eichler & 20393 & 8 & 1 & 1 & 0,0033 & 0,24 \\
\hline C. obliqua Sprengel & 20350 & 11 & 1 & 1 & 0,0033 & 0,24 \\
\hline C. sylvestris Swartz & 20394 & 18 & 8 & 3 & 0,1804 & 1,25 \\
\hline Xylosma ciliatifolia (Clos) Eichler & 20352 & 12 & 4 & 4 & 0,0164 & 0,95 \\
\hline \multicolumn{7}{|l|}{ SAPINDACEAE } \\
\hline Allophylus edulis (A. St.-Hil.) Radlk. & 20395 & 18 & 30 & 9 & 0,3636 & 3,57 \\
\hline Matayba guianensis Aublet & 20396 & 32 & 45 & 14 & 0,7922 & 6,00 \\
\hline \multicolumn{7}{|l|}{ SAPOTACEAE } \\
\hline Chrysophyllum ferrugineum C.F. Gaertner & 20437 & 13 & 2 & 2 & 0,0118 & 0,49 \\
\hline \multicolumn{7}{|l|}{ SOLANACEAE } \\
\hline Solanum bullatum Vell. & 20401 & 13 & 1 & 1 & 0,0114 & 0,25 \\
\hline S. granuloso-leprosum Dunal & 20400 & 13 & 3 & 3 & 0,0299 & 0,75 \\
\hline S. pseudoquina A. St.-Hil. & 20402 & 15 & 5 & 3 & 0,0833 & 0,94 \\
\hline S. sanctaecatharinae Dunal & 20403 & 6 & 4 & 1 & 0,0109 & 0,35 \\
\hline \multicolumn{7}{|l|}{ STYRACACEAE } \\
\hline Styrax leprosum Hooker et Arnott & 20435 & 24 & 50 & 13 & 0,7326 & 5,84 \\
\hline \multicolumn{7}{|l|}{ SYMPLOCACEAE } \\
\hline Symplocos tenuifolia Brand & 20389 & 13 & 7 & 4 & 0,0527 & 1,14 \\
\hline \multicolumn{7}{|l|}{ PENTAPHYLLACACEAE } \\
\hline Ternstroemia brasiliensis Cambess. & 20404 & 10 & 2 & 2 & 0,0187 & 0,50 \\
\hline \multicolumn{7}{|l|}{ URTICACEAE } \\
\hline Boehmeria caudata Swartz & 20407 & 6 & 1 & 1 & 0,0078 & 0,25 \\
\hline Cecropia glaziovii Snethl. & 20408 & 15 & 4 & 2 & 0,1245 & 0,80 \\
\hline \multicolumn{7}{|l|}{ VERBENACEAE } \\
\hline Aegyphila sellowiana Cham. & 20409 & 27 & 2 & 2 & 0,0705 & 0,61 \\
\hline Duranta plumere L. & 20410 & 30 & 6 & 3 & 0,3012 & 1,45 \\
\hline VOCHYSIACEAE & & & & & & \\
\hline Vochysia tucanorum Mart. & 20411 & 6 & 1 & 1 & 0,0024 & 0,23 \\
\hline WINTERACEAE & & & & & & \\
\hline Drimys brasiliensis Miers & 20412 & 11 & 11 & 6 & 0,0447 & 1,64 \\
\hline Total & & 90 & 2982 & 25 & 45,3613 & 300,00 \\
\hline
\end{tabular}


892 Loures, Carvalho, Machado \& Marques: Florística, estrutura e características do solo de um fragmento de floresta...
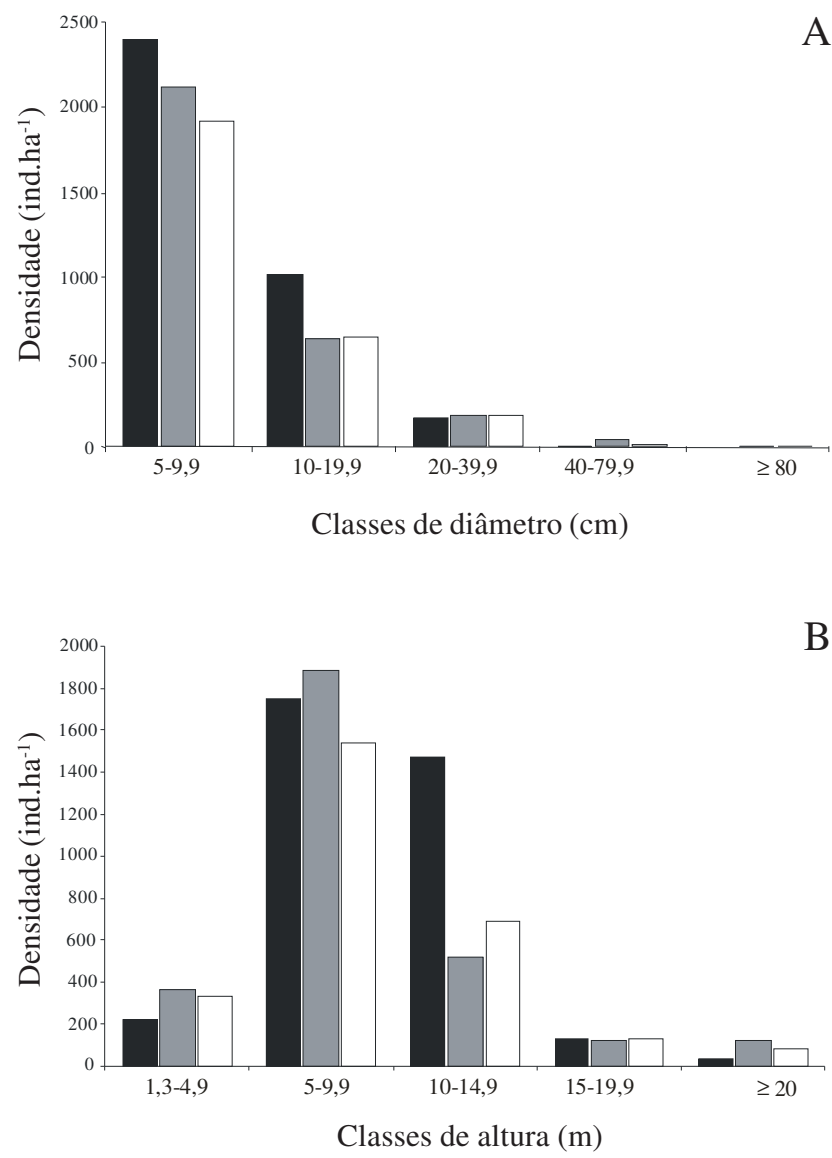

Figura 2. Distribuição da densidade por hectare de árvores com $\mathrm{DAP} \geq 5 \mathrm{~cm}$, em três tipos solos, agrupadas em classes de diâmetro (A) e de altura (B) em 1 ha de floresta estacional semidecidual montana ribeirinha, Santa Rita de Caldas, Minas Gerais. ( $\mathbf{\square}$ = Organossolos; $\mathbf{\square}=$ Gleissolos Melânicos; $\square=$ Gleissolos Háplicos).

solo. As proporções de indivíduos arbóreos não se distribuíram de forma independente dos habitas de solo do fragmento (Tab. 3). Com base na altura dos indivíduos das espécies dominantes a floresta estudada apresenta um estrato inferior, composto de Myrceugenia ovata, Ocotea laxa, Drimys brasiliensis, Rollinia emarginata, Inga vulpina, Dicksonia sellowiana; um estrato médio e superior, composto de Myrcia laruotteana, Ilex brasiliensis, Myrcia splendens, Nectandra nitidula, Myrsine leuconeura, Citronella gongonha, Sebastiania brasiliensis e Styrax leprosum e um estrato de espécies emergentes composto de Tabebuia umbellata, Cedrela odorata, Matayba guianensis, Erythrina falcata, Tapirira obtusa, Sapium glandulosum e Luehea divaricata.

Distribuição das espécies - As variáveis ambientais mais fortemente correlacionadas com o primeiro eixo da análise de correspondência canônica foram argila e cota e, com o segundo eixo, Ca e desnível (Tab. 3). As correlações entre parcelas e ambiente retratam a formação de três grupos distintos (Fig. 3A): o primeiro, formado de parcelas em solos G. Háplicos, com cotas menores e pouca drenagem, composto das seguintes espécies correlacionadas: Dicksonia sellowiana, Ilex paraguariensis, Piptocarpha macropoda e Tabebuia umbellata (Fig. 3B); o segundo grupo é influenciado por cotas altas, maior teor de matéria orgânica (Organossolos), tendo como plantas correlacionadas Talauma ovata, Tapirira obtusa, Protium spruceanum e Dendropanax cuneatum; o terceiro grupo sofre influência do desnível e $\mathrm{Ca}$ (G. Melânicos), tendo como plantas correlacionadas as generalistas Allophylus edulis, Sapium glandulosum, Guetarda uruguensis e Eugenia dodonaeifolia. Plantas importantes no caráter abundância, como Myrsine leuconeura, Myrceugenia ovata, Sebastiania brasiliensis e Citronella gongonha ficaram posicionadas próximo à divergência dos eixos das variáveis, indiferentes às variações ambientais estudadas. A espécie que apresentou maior plasticidade foi Myrceugenia ovata que esteve presente em 25 parcelas, seguida de Sebastiania brasiliensis e Nectandra nitidula (em 24), Myrcia laruotteana e Myrsine leuconeura (em 23), Citronella gongonha e Cedrela odorata (em 21) e Ilex brasiliensis (em 19).

Tabela 3. Análise de correspondência canônica (CCA): correlações internas ('intraset') nos dois primeiros eixos de ordenação e matriz de correlações ponderadas para as variáveis ambientais utilizadas na análise. Correlações com valores absolutos de p >0,5 estão indicadas em negrito.

\begin{tabular}{|c|c|c|c|c|c|c|}
\hline \multirow[t]{2}{*}{ Variáveis ambientais } & \multicolumn{2}{|c|}{ Correlações } & \multicolumn{4}{|c|}{ Variáveis ambientais } \\
\hline & Eixo 1 & Eixo 2 & $\mathrm{Ca}^{2+}$ & Argila & Cota & Desnível \\
\hline $\mathrm{Ca}^{2+}$ & 0,19 & $-0,52$ & 1 & & & \\
\hline Argila & $-0,57$ & 0,17 & $-0,39$ & 1 & & \\
\hline Cota média & 0,92 & $-0,23$ & 0,37 & $-0,57$ & 1 & \\
\hline Desnível & $-0,3$ & $-0,47$ & 0,26 & 0,17 & $-0,1$ & 1 \\
\hline
\end{tabular}



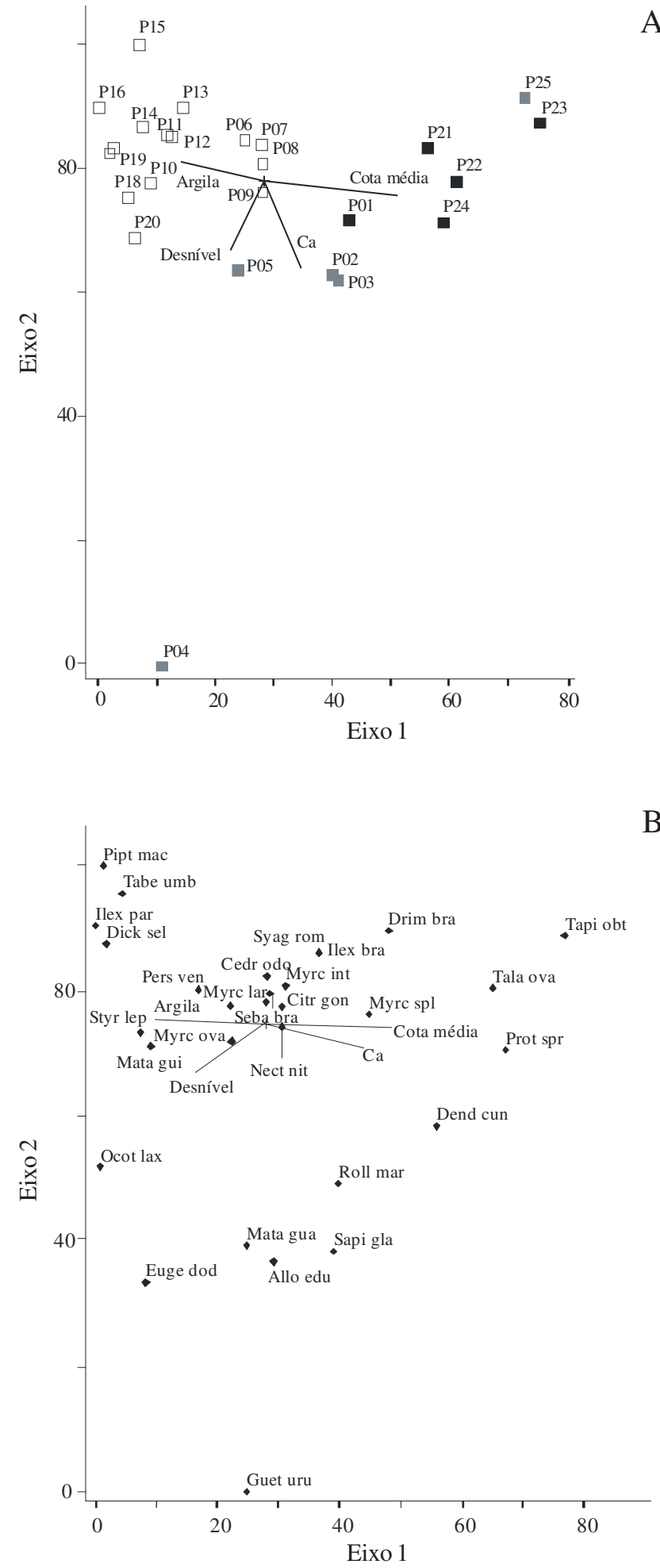

Figura 3. Análise de correspondência canônica: diagrama de ordenação das parcelas (A) e espécies (B) baseada na distribuição do número de indivíduos de 28 espécies em 1 ha de floresta estacional semidecidual montana ribeirinha, Santa Rita de Caldas, MG, e sua correlação com as cinco variáveis ambientais utilizadas (retas). As parcelas são identificadas conforme a Fig. 1 e classificadas em três tipos de solos. Os nomes das espécies estão abreviados (q.v. nomes completos na Tab. 2). ( $\mathbf{\square}=$ Organossolos; - Gleissolos Melânicos; $\square=$ Gleissolos Háplicos).

\section{A Discussão}

Perfil e contexto florístico - A família Myrtaceae que aparece em primeiro lugar em número de espécies no presente estudo, também o foi nas florestas de altitude de Bocaina de Minas (Carvalho et al. 2005) e Camanducaia (França \& Stehmann 2004). As famílias Lauraceae, Solanaceae e Asteraceae, importantes aqui, também são destaques naquelas florestas, fato este também salientado por Oliveira Filho \& Fontes (2000) para áreas de altitude. Rubiaceae e Melastomataceae que ocorrem com relativa expressão em florestas de encostas em áreas de altitude, como Camanducaia e Bocaina de Minas, com seis e doze espécies, respectivamente (França \& Stehmann 2004; Carvalho et al. 2005), não foram expressivas no presente estudo. Nas florestas de brejo do estado de São Paulo, Rubiaceae e Melastomataceae, também não foram dominantes (Toniato et al. 1998; Teixeira \& Assis 2005). A família Fabaceae, a partir de $1.500 \mathrm{~m}$ de altitude perde importância (Carvalho et al. 2005) e a floresta paludosa em estudo, a $1.200 \mathrm{~m}$, reflete essa tendência. $\mathrm{O}$ gênero Ilex foi relacionado com áreas de altitude nas regiões sudeste e sul do Brasil (França \& Stehmann 2004; Carvalho et al. 2005; Souza \& Lorenzi 2005, presente estudo). A floresta paludosa de altitude de Santa Rita de Caldas, apresentou espécies comuns com matas da região sul do Brasil, como Araucaria angustifolia, Myrcianthes gigantea, Ilex paraguariensis, Cordyline spectabilis, Dicksonia sellowiana, Styrax leprosum, Myrciaria delicatula e Solanum sanctaecatharinae (Reitz et al. 1983; Dias et al. 1998; Barddal et al. 2003). As espécies Chionanthus trichotomus e Gaylussacia brasiliensis, aqui encontradas, ainda não haviam sido citadas para o estado de Minas Gerais em áreas aluviais e paludosas. Drymis brasiliensis, previsível para áreas pantanosas de altitude na região sudeste do Brasil (Souza \& Lorenzi 2005), foi representada no presente estudo com 11 exemplares e não ocorreu em floresta paludosa de menor altitude na região (Rocha et al. 2005). Myrceugenia ovata, Ilex brasiliensis e Styrax leprosum, sobressaíram em Santa Rita de Caldas mas não ocorreram em outros fragmentos em áreas homólogas (Toniato et al. 1998; Ivanauskas et al. 1997; Marques et al. 2003; Teixeira \& Assis 2005; Rocha et al. 2005) indicando que estas espécies sejam típicas da região planáltica e serrana do sul de Minas Gerais. Salienta-se também no presente estudo a dominância de Myrsine leuconeura $(=M$. intermedia $)$ que ocorre em outros locais de 
894 Loures, Carvalho, Machado \& Marques: Florística, estrutura e características do solo de um fragmento de floresta...

forma discreta (Toniato et al. 1998; Rocha et al. 2005).

Há poucas citações em trabalhos no Brasil apontando o clima como fator determinante da florística e da estrutura de uma floresta. No estado de São Paulo, os dados disponíveis sobre matas higrófilas são de regiões semelhantes quanto às condições climáticas, situadas em altitudes entre 470 e 660 m, o que não seria fator determinante para a variação florística (Scudeller et al. 2001). Nas regiões serranas, as massas de ar frio no inverno tendem a se acumular nas baixadas, onde podem ocorrer geadas (Ometo 1981). Essa pode ser a explicação para a ausência de certas espécies, no local de estudo, e que são comuns em outras florestas ribeirinhas das regiões sul e sudeste do Brasil, como Calophyllum brasiliense Camb., Tapirira guianensis Aubl., Croton urucurana Baill., Genipa americana L., Guarea guidonea (L.) Sleum., Cytharexyllum myrianthus Cham, Euterpe edulis Mart. e Cecropia pachystachia Trec. (Rodrigues \& Nave 2000; Barddal et al. 2003; Marques et al. 2003; Budke et al. 2004; Rocha et al. 2005).

Estrutura e distribuição das espécies - A densidade de árvores na floresta paludosa do riacho Luiz Espanhol, de 2.982 ind.ha $^{-1}$, pode ser considerada alta se comparada com as florestas paludosas e florestas de altitude do sudeste do Brasil (Costa et al. 1997; Ivanauskas et al. 1997; França \& Stehmann 2004; Carvalho et al. 2005; Rocha et al. 2005; Teixeira \& Assis 2005). O índice de diversidade no local de estudo de 2,98, foi superior ao registrado em floresta em Campinas $(2,80)$ e em Itatinga $(2,60)$ (Ivanaukas et al. 1997; Toniato et al. 1998), porém inferior ao registrado em Bocaina de Minas (4,15) (Carvalho et al. 2005).

O gênero Sebastiania possui espécies peculiares e importantes em florestas paludosas (Ivanauskas et al. 1997). Neste trabalho, S. brasiliensis foi a terceira espécie em abundância, enquanto que $S$. commersoniana tem abundância seis vezes superior a da segunda colocada, em matas de aluvião de Araucária (Barddal et al. 2003). Em Santa Maria, S. brasiliensis também é uma espécie abundante (Budke et al. 2004). Nectandra nitidula é uma espécie que aqui aparece em sétimo lugar em importância e teve participação em nascentes difusas em Lavras (Pinto et al. 2005). Esta espécie se encaixaria no grupo das complementares indiferentes (conforme Torres et al. 1992), que pode ocorrer em ambientes paludosos, áreas ciliares sazonalmente inundáveis e em outras permanentemente secas. Dendropanax cuneatum e Matayba guianensis (= M. eleaegnoides) foram similares em termos estruturais e ambientais. Em duas áreas de encosta de altitude estas espécies estão ausentes (França \& Stehmann 2004; Carvalho et al. 2005) indicando que elas têm mais afinidade com ambientes ripários ou proximidades.

A ocorrência de indivíduos perfilhados ou enforquilhados no nível da superfície do solo em florestas paludosas também foi verificada por Ivanauskas et al. (1997). As causas do tombamento das plantas em ambientes paludosos podem estar relacionadas à pequena profundidade do sistema radicular em solos com lençol freático muito superficial e à instabilidade dos solos hidromórficos (MartinezRamos 1985). A percentagem de árvores mortas encontrada $(7,63 \%$ do total de indivíduos em pé - os vivos mais os mortos) é compatível com os valores de $5 \%$ a $8 \%$ encontrados em ambientes paludosos e nãopaludosos (Martins 1991; Schlittler et al. 1995; Toniato et al. 1998).

A maioria dos indivíduos amostrados $(70 \%)$ se encaixou na classe diamétrica abaixo de $10 \mathrm{~cm}$, retratando uma mata "paliteira" (árvores finas), de alta densidade. O porte menor dos indivíduos em áreas encharcadas pode também ser causado pela presença de sistema radicular superficial e instabilidade dos solos (Bianchini et al. 2003). Comparando o diâmetro dos indivíduos do local de estudo com aqueles da floresta de encosta e de altitude de Bocaina de Minas (Carvalho et al.2005), verifica-se no presente estudo um número maior de indivíduos na primeira e segunda classe diamétrica, indicando que as árvores de floresta paludosa são mais finas do que as de floresta de encosta. Também, comparando a altura dos indivíduos destas duas florestas, observa-se que a floresta de encosta apresenta maior número de árvores altas que a paludosa.

A influência da umidade dos solos na distribuição de espécies arbóreas e nas variações fisionômicas nas florestas tropicais tem largo subsídio na literatura (e.g. Basnet 1992; Oliveira Filho et al. 1994; Clark et al. 1998; Botrel et al. 2002). A proximidade da represa (cota) e o teor de argila foram as variáveis que mais contribuíram para a divisão dos grupos de parcelas e espécies em três hábitats. Em duas áreas, uma de cotas mais altas e com maior teor de matéria orgânica (Organossolos) e outra de cotas baixas com solos argilosos (G. Háplicos) e pouca drenagem, destacaram-se Talauma ovata e Dendropanax cuneatum, espécies comuns nas florestas paludosas do sudeste brasileiro (Rocha et al. 2005), além de Dicksonia sellowiana e Tabebuia umbellata, 
espécies peculiares exclusivas de áreas paludosas (Lorenzi 1998, Carvalho et al. 2005). Numa terceira área, intermediária e menos úmida (G. Melânicos), ocorreram as espécies complementares indiferentes. A despeito do presente trabalho haver detectado uma clara correlação entre a distribuição de espécies e as características do substrato, com ênfase para a disponibilidade de água nos solos, foi também identificado um razoável montante de variância não explicada pelas variáveis estudadas. O histórico de perturbação diferenciada é uma possível variável não considerada e de difícil controle. Se perturbações naturais promovidas pelo processo de silvigênese podem criar um mosaico de eco-unidades de variada composição e estrutura (Oldeman 1983), aquelas promovidas pelo homem e pelo gado constituem um fator adicional a ser considerado na área de estudo (Toniato \& Oliveira Filho 2004). Acrescente-se a isto que ainda se pode deixar uma larga margem para efeitos estocásticos na distribuição das espécies.

\section{Agradecimentos}

Aos professores Ary Teixeira de Oliveira Filho (UFLA) e Marcos Sobral (UFMG), pelo auxílio nas identificações botânicas; Lúcia Ferreira, Wellington Fleming e Edson Pisteli (EAFI), pelos levantamentos topográficos e análises de solos.

\section{Referências bibliográficas}

APG II. 2003. An update of the Angiosperm Phylogeny Group classification for the orders and families of flowering plants: APG II. Botanical Journal of the Linnean Society 141: 399-436.

Barddal, M.L.; Roderjan, C.V.; Galvão, F. \& Curcio, G.R. 2003. Caracterização florística e fitossociológica de um trecho sazonalmente inundável de floresta aluvial, em Araucária, PR. Ciência Florestal 14: 37-50.

Basnet, K. 1992. Effect of topography on the pattern of trees in tabonuco (Dacryodes excelsa) dominated rain forest of Puerto Rico. Biotropica 24: 31-42.

Bianchini, E.; Popolo, RS.; Dias, M.C; Pimenta, J.A. Diversidade e estrutura de espécies arbóreas em área alagável do município de Londrina, sul do Brasil. Acta Botanica Brasilica 17: 405-419.

Botrel, R.T.; Oliveira Filho A.T.; Rodrigues, L.A. \& Curi, N. 2002. Influência do solo e topografia sobre as variações da composição florística e estrutura da comunidade arbóreo-arbustiva de uma floresta estacional semidecidual em Ingaí, MG. Revista Brasileira de Botânica 25: 195-213.

Brower, J.E. \& Zar, J.H. 1984. Field and laboratory methods for general ecology. Dubuque, W.M.C. Brow.
Budke, J.C.; Giehl, E.L.H.; Athaide, E.A.; Eisinger, S.M. \& Záchia, R.A. 2004. Florística e fitossociologia do componente arbóreo de uma floresta ribeirinha, arroio Passo das Tropas, Santa Maria, RS, Brasil. Acta Botanica Brasilica 18: 581-589.

Carvalho, D.A.; Oliveira Filho, A.T.; van den Berg, E.; Fontes, M.L; Vilela, E.A.; Melo Marques, J.J.G.S. \& Carvalho, W.A.C. 2005. Variações florísticas e estruturais do componente arbóreo de uma floresta ombrófila altomontana às margens do Rio Grande, Bocaina de Minas, MG, Brasil. Acta Botanica Brasilica 19: 91-109.

Clark, D.B.; Clark, D.A. \& Read, J.M. 1998. Edaphic variation and the mesoscale distribution of tree species in a neotropical rain forest. Journal of Ecology 86: 101-112.

Costa, F.R.C.; Schlittler, F.H.M.; Cesar, O. \& Monteiro, R. 1997. Aspectos florísticos e fitossociológicos de um remanescente de mata de brejo no município de Brotas, SP. Arquivos de Biologia e Tecnologia 40:263-270.

Dias, M.C.; Vieira, A.O.S.; Nakajima, J.N.; Pimenta, J.A. \& Lobo, P.C. 1998. Composição florística do componente arbóreo das florestas ciliares do rio Iapó, na bacia do rio Tibagi, Tibagi, PR. Revista Brasileira de Botânica 21: 183-195.

EMBRAPA. 1997. Manual de métodos de análise de solo. 2.ed. Rio de Janeiro, Empresa Brasileira de Pesquisa agropecuária, Centro Nacional de Pesquisa de Solos.

EMBRAPA. 1999. Sistema brasileiro de classificação de solos. Rio de Janeiro, Empresa Brasileira de Pesquisa Agropecuária, Centro Nacional de Pesquisa de Solos.

França, G.S. \& Stehmann, J.R. 2004. Composição florística e estrutura do componente arbóreo de uma floresta altimontana no município de Camanducaia, Minas Gerais, Brasil. Revista Brasileira de Botânica 27: 19-30.

Gibbs, P.E.; Leitão Filho, H.F. \& Abbott, R.J. 1980. Application of the pointcentred quarter method in floristic survey of an área of gallery forest at MogiGuaçu, SP, Brasil. Revista Brasileira de Botânica 3: 17-22.

IBGE. 1997. Geografia do Brasil: Região Sudeste. Rio de Janeiro, Instituto Brasileiro de Geografia e Estatística.

INDI. 1977. Informações básicas para investidores. Belo Horizonte, Instituto de Desenvolvimento Industrial, Sul de Minas.

Ivanauskas, N.M.; Rodrigues, R. \& Nave, G. 1997. Fitossociologia e seletividade de espécies numa floresta de brejo em Itatinga, SP. Revista Brasileira de Botânica 20: $139-153$.

Joly, C.A. 1986. Heterogeneidade ambiental e diversidade de estratégias adaptativas de espécies arbóreas de mata de galeria. Pp. 19-38. In: Anais do $10^{\circ}$ Simpósio da Academia de Ciências de São Paulo: perspectivas da ecologia teórica. São Paulo, Aciesp.

Kent, M. \& Coker, P. 1992. Vegetation, description and analysis, a pratical approach. London, Belhaven.

Leitão Filho, H. F. 1982. Aspectos taxonômicos das florestas do Estado de São Paulo. Silvicultura em São Paulo 16A: 197-206.

Lima, W.P. 1989. Função hidrológica da mata ciliar. Pp. 25-42. In: L.M. Barbosa (coord.). Anais do I Simpósio sobre Mata Ciliar. Campinas, Fundação Cargil. 
896 Loures, Carvalho, Machado \& Marques: Florística, estrutura e características do solo de um fragmento de floresta...

Lorenzi H. 1998. Árvores Brasileiras: manual de identificação e cultivo de plantas arbóreas nativas do Brasil. v.1. Nova Odessa, Plantarum.

Marques, M.C.M.; Silva, S.M. \& Salino, A. 2003. Florística e estrutura do compomente arbustivo-arbóreo de uma floresta higrófila da bacia do rio Jacaré-Pepira. Acta Botanica Brasilica 17: 495-506.

Martinez-Ramos, M. 1985. Claros, ciclos vitales de los árboles tropicales y regeneración natural de las selvas altas perenifolias. Pp. 191-239. In: A. Gomez-Pómpa \& S.R. Amo (eds.). Investigaciones sobre la regeneración de selvas altas em Veracruz, México. México, Editorial Alhambra Mexicana.

Martins, F.R. 1991. Estrutura de uma floresta mesófila. Campinas, EDUNICAMP.

Oldeman, R.A.A. 1983. Tropical rain forest: architecture, sylvigenesis and diversity. Pp. 139-150. In: S.L. Sutton; T.C. Whitmore \& A.C. Chadwick (eds.). Tropical rain forest: ecology and management. Oxford, Blackwell.

Oliveira Filho, A.T. \& Fontes, M.A.L. 2000. Patterns of floristic differentiation among Atlantic forest in southeastern Brazil and the influence of climate. Biotropica 32: 793-810.

Oliveira Filho, A.T. \& Ratter, J.A. 1994. Database: Woody flora of $\mathbf{1 0 6}$ forest areas of eastern tropical South America. Edinburgh, Royal Botanic Garden.

Oliveira Filho, A.T.; Vilela, E.A.; Carvalho, D.A. \& Gavilanes, M.L. 1994. Effects of soils and topography on the distribution of tree species in a tropical riverine forest in south-eastern Brazil. Journal of Tropical Ecology 10: 483-508.

Ometo, J.K. 1981. Bioclimatologia vegetal. São Paulo,Editora Agronômica Ceres.

Paschoal, M.E.S. \& Cavassan, O. 1999. A flora arbórea da mata de brejo de Ribeirão Peliantra, Agudos-SP. Naturalia 4: 171-191.

Pedralli, G. \& Teixeira, M.C.B. 1997. Reservatórios: alternativas para proteção das comunidades vegetais na sua área de influência. Ensaios e Dissertações 5: 34-41.

Pinto, L.V.A.; Botelho, S.A.; Oliveira Filho, A.T. \& Davide, A.C. 2005. Estudo da vegetação com subsídios para propostas de recuperação das nascentes da bacia hidrográfica do Ribeirão Santa Cruz, Lavras, MG. Revista Árvore 29: 775-794.

Reitz, R.; Klein, R.M.; Reis, A. 1983. Projeto Madeira do Rio Grande do Sul. Sellowia 34/35: 5-483.

Rocha, C.T.V.; Carvalho, D.A.; Fontes, M.A.; Oliveira Filho, A.T.; van den Berg, E. \& Marques, J.J.G.S. 2005. Comunidade arbórea de um continuum entre floresta paludosa e de encosta em Coqueiral, MG. Revista Brasileira de Botânica 28: 203-217.
Rodrigues, R.R. \& Nave, A.G. 2000. Hetrogeneidade florística das matas ciliares. Pp. 45-71. In: R.R. Rodrigues \& H.F. Leitão Filho (eds.). Matas ciliares. São Paulo, EDUSP.

Rodrigues, R.R. \& Shepherd, G.J. 1993. Considerações sobre os fatores atuantes em matas ciliares e condições ecológicas dominantes na faixa ciliar do sudeste brasileiro. In: Curso sobre recuperação de áreas degradadas. Curitiba, UFP.

Schlittler, F.H.M.; Marinis, G. \& Cesar, O. 1995. Estudos fitossociológicos na floresta do Morro do Diabo (Pontal do Paranapanema, SP, Brasil). Arquivos de Biologia e Tecnologia 38: 217-234.

Scudeller, V.V.; Martins, F.R. \& Shefherd, G.J. 2001. Distribution and abundance of arboreal species in the atlantic ombrophilous dense forest in Southeastern Brazil. Plant Ecology 152: 185-199.

Souza, V.C. \& Lorenzi, H. 2005. Botânica sistemática. Nova Odessa, Instituto Plantarum.

Teixeira, A.P. \& Assis, M.A. 2005. Caracerização florística e fitossociológica do componente arbustivo-arbóreo de uma floresta paludosa no Município de Rio Claro (SP), Brasil. Revista Brasileira de Botânica 28: 467-476.

ter Braak, C.J.F. 1987. The analysis of vegetationenvironment relationship by canonical correspondence analysis. Vegetatio 69: 69-77.

Toniato, M.T.Z. \& Oliveira A.T. 2004. Variations in tree community composition and structure in a fragment of tropical semideciduous forest in southeastern Brazil related to different human disturbance histories. Forest Ecology and Management 198: 319-339.

Toniato, M.T.Z.; Leitão Filho, H.F. \& Rodrigues, R.R. 1998. Fitossociologia de um remanescete de floresta higrófila (mata de brejo) em Campinas, SP. Revista Brasileira de Botânica 21: 197-210.

Torres, R.B.; Matthes, L.A.F.; Rodrigues R.R. \& Leitão, H.F. 1992. Espécies florestais nativas para plantio em áreas de brejo. O Agonômico 44: 6-13.

Torres, R.B.; Mathes, L.A. \& Rodrigues, R.R. 1994. Florística e estrutura do componente arbóreo de mata de brejo em Campinas, SP. Revista Brasileira de Botânica 17: 189-194.

Veloso, H.P.; Rangel Filho, A.L.R. \& Lima, J.C.A. 1991. Classificação da vegetação brasileira adaptada a um sistema universal. Rio de janeiro, Instituto Brasileiro de Geografia e Estatística.

Zar, J.H. 1996. Biostatistical analysis, 3. ed. New Jersey, Prentice Hall. 